TRANSACTIONS OF THE

AMERICAN MATHEMATICAL SOCIETY

Volume 349, Number 10, October 1997, Pages 3977-3987

S 0002-9947(97)01716-9

\title{
THE WILLS CONJECTURE
}

\author{
NOAH SAMUEL BRANNEN
}

\begin{abstract}
Two strengthenings of the Wills conjecture, an extension of Bonnesen's inradius inequality to $n$-dimensional space, are obtained. One is the sharpest of the known strengthenings of the conjecture in three dimensions; the other employs techniques which are fundamentally different from those utilized in the other proofs.
\end{abstract}

One of the best known geometric inequalities is the isoperimetric inequality, which states that of all closed planar curves with fixed perimeter, a circle encloses the greatest area. If $K$ is a planar convex body (a convex "body" is a compact convex set with nonempty interior) of perimeter $L$ with area $A$, then this inequality can be expressed as

$$
L^{2}-4 \pi A \geq 0
$$

with equality only when the boundary of $K$ is a circle.

A strengthening of (1) was given by the Danish mathematician T. Bonnesen, who demonstrated in 1929 (see [5]) that if $K$ has circumradius $R$ and inradius $r$, then

$$
L^{2}-4 \pi A \geq \pi^{2}(R-r)^{2}
$$

This inequality can be derived from the inequality

$$
0 \geq A-\lambda L+\lambda^{2} \pi, \quad r \leq \lambda \leq R .
$$

The inequality (3) with $\lambda=r$ is known as Bonnesen's inradius inequality.

J. M. Wills [15] conjectured in 1970 that

$$
0 \geq V-r S+(n-1) r^{n} \omega_{n},
$$

which would be an extension of Bonnesen's inradius inequality to higher dimensions. Here $V$ is the $n$-dimensional volume of a convex body in $\Re^{n}, r$ is its inradius, $S$ is its $n$-dimensional surface area and $\omega_{n}$ is the volume of the $n$-dimensional unit ball. This result was proven simultaneously in 1973 by J. Bokowski [2] and V. I. Diskant [7]. Both proofs demonstrated that the Wills conjecture is a consequence of a result due to $\mathrm{H}$. Hadwiger [8], namely

$$
\left(\frac{1}{n}\right)^{\frac{n}{n-1}} S^{\frac{n}{n-1}}-\omega_{n}^{\frac{1}{n-1}} V \geq\left[\left(\frac{1}{n}\right)^{\frac{1}{n-1}} S^{\frac{1}{n-1}}-r \omega_{n}^{\frac{1}{n-1}}\right]^{n}
$$

Received by the editors February 9, 1995 and, in revised form, August 15, 1995.

1991 Mathematics Subject Classification. Primary 52A40.

Key words and phrases. Circumradius, convex body, inner parallel body, inradius, mixed volume, quermassintegral. 
In 1979 Robert Osserman [10] showed that

$$
0 \geq V-r S+(n-1) r^{n} \omega_{n}\left(\frac{S}{n r^{n-1} \omega_{n}}\right)^{\frac{n-2}{n-1}},
$$

which is a strengthening of the Wills conjecture. He derived this result from (5). In 1988 Jane Sangwine-Yager [11] derived an even stronger result,

$0 \geq V-r S+(n-1) r^{2} W_{2}+(n-1)(n-2) \int_{0}^{r}(r-s) V\left(K_{-s}, \ldots, K_{-s}, K_{-r}, B, B\right) d s$,

where $W_{2}$ is the second quermassintegral of $K$. Here $K_{-s}$ and $K_{-r}$ are inner parallel bodies of $K$, and $V\left(K_{-s}, \ldots, K_{-s}, K_{-r}, B, B\right)$ is a mixed volume. The pertinent definitions will be given subsequently. Sangwine-Yager derived her result as a corollary to a lower bound on the volume of inner parallel bodies. We shall give another lower bound on the volume of inner parallel bodies. This result has as a corollary

$$
0 \geq V-r S+n \sum_{i=0}^{n-2} \int_{0}^{r} t V(\underbrace{K_{-t}, \ldots, K_{-t}}_{i}, K, \ldots, K, B, B) d t,
$$

a strengthening of the Wills conjecture which is a sharper inequality in $\Re^{3}$ than the inequality given by Sangwine-Yager. It also provides the shortest proof of the Wills conjecture that we have been able to find. An additional strengthening of the Wills conjecture is

$$
0 \geq V-r S+\left[n \sum_{i=0}^{n-2} \frac{i}{i+1}\left(\begin{array}{c}
n-1 \\
i
\end{array}\right) r^{i+1} W_{i+1}\left(K_{-r}\right)\right]+(n-1) r^{n} \omega_{n}
$$

where $W_{i+1}$ is the $(i+1)$ st quermassintegral. This strengthening results from taking an approach which is fundamentally different than the methods employed in the other proofs.

We begin with some definitions. If $K$ and $L$ are convex bodies, their Minkowski sum $K+L$ is defined to be $\{k+l: k \in K, l \in L\}$. If $\lambda$ is a scalar then $\lambda K$ is $\{\lambda k: k \in K\}$. If the convex body $K$ in $\Re^{n}$ is a Minkowski linear combination of $m$ convex bodies, i.e. $K=\lambda_{1} K_{1}+\lambda_{2} K_{2}+\ldots+\lambda_{m} K_{m}, \lambda_{1}, \ldots, \lambda_{m} \geq 0$, then the volume of $K$ can be expressed as an $n$th degree homogeneous polynomial in the $\lambda_{i}$ as follows:

$$
V(K)=\sum_{1 \leq p_{1}, \ldots, p_{n} \leq m} V\left(K_{p_{1}}, K_{p_{2}}, \ldots, K_{p_{n}}\right) \lambda_{p_{1}} \lambda_{p_{2}} \ldots \lambda_{p_{n}} .
$$

Here the summation is extended over all $p_{i}$ independently as $i$ varies from 1 to $n$ (see [13], page 275$)$. The coefficients $V\left(K_{p_{1}}, K_{p_{2}}, \ldots, K_{p_{n}}\right)$ are called "mixed volumes".

The mixed volume $V(\underbrace{K, \ldots, K}_{n-i}, \underbrace{B, \ldots, B}_{i})$ with $i$ copies of the unit ball $B$ in $\Re^{n}$

will be denoted by $W_{i}(K)$ and is called the $i$ th quermassintegral of $K$. It can be shown that

$$
\begin{gathered}
W_{0}(K)=V(K), \\
n W_{1}(K)=S(K), \\
\frac{2}{\omega_{n}} W_{n-1}(K)=\bar{M}(K),
\end{gathered}
$$


where $\bar{M}(K)$ is the mean width of $K$, and

$$
W_{n}(K)=\omega_{n} .
$$

More generally, for a fixed convex body $E$ we define

$$
W_{i}(K ; E)=V(\underbrace{K, \ldots, K}_{n-i}, \underbrace{E, \ldots, E}_{i})
$$

to be the $i$ th relative quermassintegral of $K$.

Also, we define the relative inradius of $K$ with respect to $E$ to be

$$
r(K ; E)=\sup \{r: \text { some translate of } r E \subseteq K\},
$$

and the relative surface area of $K$ with respect to $E$ to be

$$
S(K ; E)=n W_{1}(K ; E) .
$$

Mixed volumes have several interesting properties. We present four properties which will be used in the strengthenings of the Wills conjecture.

Property 1. $V(*, *, \ldots, *)$ is symmetric in its arguments.

Property 2. $V(*, *, \ldots, *)$ is a Minkowski multilinear functional.

Property 3. $V(*, *, \ldots, *)$ is monotone, in the sense that if $K_{1} \subseteq K_{1}^{\prime}$, then

$$
V\left(K_{1}, K_{2}, \ldots, K_{n}\right) \leq V\left(K_{1}^{\prime}, K_{2}, \ldots, K_{n}\right) .
$$

Property 4. If $K_{1}, \ldots, K_{n}$ are convex bodies in $\mathfrak{R}^{n}$ and $m<n$ is a natural number, then

$$
V\left(K_{1}, \ldots, K_{n}\right)^{m} \geq \prod_{i=1}^{m} V(\underbrace{K_{i}, \ldots, K_{i}}_{m}, K_{m+1}, \ldots, K_{n}) .
$$

The above inequality is due to Alexandrov [1]. Proofs of the first three properties can be found in [13], pages 272-279.

The following formula concerning the quermassintegrals of Minkowski sums of convex bodies will be used in a strengthening of the Wills conjecture.

If $K$ and $L$ are convex bodies in $\Re^{n}, E$ is a fixed convex body in $\Re^{n}$ and $s \geq 0$ and $t \geq 0$ are scalars, then the $i$ th relative quermassintegral of $s K+t L$ satisfies

$$
W_{i}(s K+t L)=\sum_{j=0}^{n-i}\left(\begin{array}{c}
n-i \\
j
\end{array}\right) s^{n-i-j} t^{j} V(\underbrace{K, \ldots, K}_{n-i-j}, \underbrace{L, \ldots, L}_{j}, \underbrace{E, \ldots, E}_{i}) .
$$

This formula is a generalization of a result known as Steiner's formula (Steiner demonstrated this result only for the case $n=3$ ), which is

$$
V(K+\lambda B)=\sum_{i=0}^{n}\left(\begin{array}{c}
n \\
i
\end{array}\right) \lambda^{i} W_{i}(K) .
$$

We turn our attention now to inner parallel bodies. In the following, all our considerations will be restricted to $n$-dimensional Euclidean space $\Re^{n}, n \geq 2$.

The outer parallel body at a distance $\lambda$ of a convex body $K$ will be denoted by $K_{\lambda}=K+\lambda B$, where $B$ is the unit ball. The inner parallel body of $K$ at a distance $\lambda$, which is the set of all points of $K$ whose distance from the boundary of $K$ is at least $\lambda$, will be denoted by $K_{-\lambda}$. 
We define the relative inner parallel body of $K$ with respect to a fixed convex body $E$ at a distance $\lambda, 0 \leq \lambda \leq r(K ; E)$, to be

$$
K_{-\lambda(E)}=\{x: \quad x+\lambda E \subseteq K\} .
$$

Once the convex body $E$ is fixed we will denote the relative inner parallel body simply by $K_{-\lambda}$.

The next two lemmas will be used often in what follows. The first lemma is a direct consequence of the above definition.

Lemma 1. Let $E$ be a fixed convex body and let $K$ be any convex body in $\Re^{n}$. Let the relative inradius of $K$ be $r$. For each $\lambda, 0 \leq \lambda \leq r$,

$$
K_{-\lambda}+\lambda E \subseteq K
$$

Lemma 2. Under the same assumptions as in the previous lemma, for each $\lambda$, $0 \leq \lambda \leq r$, let $S\left(K_{-\lambda}\right)$ be the relative surface area of $K_{-\lambda}$. Then

$$
V(K)=\int_{0}^{r} S\left(K_{-\lambda}\right) d \lambda
$$

The preceding lemma, as well as the next, is due to Bol [4].

Lemma 3. $\left[W_{n-i}\left(K_{-t(E)}, E\right)\right]^{\frac{1}{i}}$ is a concave function of $t$ for $i=0, \ldots, n-1$.

We will now give two lower bounds and one upper bound for the volume of relative inner parallel bodies. The two lower bounds will be compared.

Theorem 1. (Sangwine-Yager) If $E$ is a fixed convex body and $K$ is any convex body in $n$-dimensional Euclidean space $\Re^{n}$ and $K$ has volume $V$, relative surface area $S$, relative second quermassintegral $W_{2}$, and relative inradius $r$, then for all $\lambda$, $0 \leq \lambda \leq r$

$$
\begin{aligned}
V\left(K_{-\lambda}\right) \geq & V-\lambda S+(n-1) \lambda^{2} W_{2} \\
& +(n-1)(n-2) \int_{0}^{\lambda}(\lambda-s) V\left(K_{-s}, \ldots, K_{-s}, K_{-\lambda}, E, E\right) d s .
\end{aligned}
$$

This lower bound for the volume of the relative inner parallel body at a distance $\lambda$ is due to Sangwine-Yager [11].

What follows is a general result on the relative quermassintegrals of relative inner parallel bodies.

Theorem 2. If $E$ is a fixed convex body and $K$ is any convex body in $\Re^{n}$ with ith relative quermassintegral $W_{i},(i+1)$ th relative quermassintegral $W_{i+1}$ and relative inradius $r$, then for all $0 \leq \lambda \leq r$, we have

$$
\begin{aligned}
W_{i}\left(K_{-\lambda}\right) \leq & W_{i}-\lambda W_{i+1}-\lambda W_{i+1}\left(K_{-\lambda}\right) \\
& -\lambda \sum_{k=1}^{n-i-2} V(\underbrace{K_{-\lambda}, \ldots, K_{-\lambda}}_{k}, \underbrace{K, \ldots, K}_{n-k-i-1}, \underbrace{E, \ldots, E}_{i+1}) .
\end{aligned}
$$


Proof. By Lemma $1, K \supseteq K_{-\lambda}+\lambda E$ for all $0 \leq \lambda \leq r$. Using this fact and the properties of mixed volumes, we have

$$
\begin{gathered}
W_{i}=V(\underbrace{K, \ldots, K}_{n-i}, \underbrace{E, \ldots, E}_{i}) \geq V(K_{-\lambda}+\lambda E, \underbrace{K, \ldots, K}_{n-i-1}, \underbrace{E, \ldots, E}_{i}) \\
=V(K_{-\lambda}, \underbrace{K, \ldots, K}_{n-i-1}, \underbrace{E, \ldots, E}_{i})+\lambda W_{i+1} \\
=V(K_{-\lambda}, K_{-\lambda}+\lambda E, \underbrace{K, \ldots, K}_{n-i-2}, \underbrace{E, \ldots, E}_{i})+\lambda W_{i+1} \\
=V(K_{-\lambda}, K_{-\lambda}, \underbrace{K, \ldots, K}_{n-i-2}, \underbrace{E, \ldots, E}_{i})+\lambda V(K_{-\lambda}, \underbrace{K, \ldots, K}_{n-i-2}, \underbrace{E, \ldots, E}_{i+1})+\lambda W_{i+1} \\
\ldots \geq W_{i}\left(K_{-\lambda}\right)+\lambda W_{i+1}+\lambda W_{i+1}\left(K_{-\lambda}\right)+\lambda \sum_{k=1}^{n-i-2} V(\underbrace{K_{-\lambda}, \ldots, K}_{k}, \underbrace{K, \ldots, K}_{n-k-i-1} \underbrace{E, \ldots, E}_{i+1}) .
\end{gathered}
$$

This completes the proof.

With $S(K)$ denoting the relative surface area of $K$ with respect to $E$, we have the following corollaries.

Corollary 1. When $i=0$ Theorem 2 becomes

$V\left(K_{-\lambda}\right) \leq V(K)-\lambda \frac{1}{n} S(K)-\lambda \frac{1}{n} S\left(K_{-\lambda}\right)-\lambda \sum_{k=1}^{n-2} V(\underbrace{K_{-\lambda}, \ldots, K_{-\lambda}}_{k}, \underbrace{K, \ldots, K}_{n-k-1}, E)$,

which gives us an upper bound for the volume of $K_{-\lambda}$.

The next corollary gives us a lower bound for the volume of $K_{-\lambda}$.

Corollary 2. For all $0 \leq \lambda \leq r$ and for all $n>2$,

$$
\begin{aligned}
V\left(K_{-\lambda}\right) \geq & V(K)-\lambda S(K)+n \frac{\lambda^{2}}{2} W_{2}(K) \\
& +n \sum_{k=1}^{n-2} \int_{0}^{\lambda} t V(\underbrace{K_{-t}, \ldots, K_{-t}}_{k}, \underbrace{K, \ldots, K}_{n-k-2}, E, E) d t .
\end{aligned}
$$

Proof. When $i=1$ Theorem 2 becomes

$$
\begin{aligned}
\frac{1}{n} S\left(K_{-\lambda}\right) \leq & \frac{1}{n} S(K)-\lambda W_{2}(K)-\lambda W_{2}\left(K_{-\lambda}\right) \\
& -\lambda \sum_{k=1}^{n-3} V(\underbrace{K_{-\lambda}, \ldots, K_{-\lambda}}_{k}, \underbrace{K, \ldots, K}_{n-k-2}, E, E) .
\end{aligned}
$$

Integrating both sides from 0 to $t$ with respect to $\lambda$ and applying Theorem 2, we obtain

$$
\begin{aligned}
& \frac{1}{n}\left[V(K)-V\left(K_{-t}\right)\right] \\
& \quad \leq t \frac{1}{n} S(K)-\frac{t^{2}}{2} W_{2}(K)-\sum_{k=1}^{n-2} \int_{0}^{t} \lambda V(\underbrace{K_{-\lambda}, \ldots, K_{-\lambda}}_{k}, \underbrace{K, \ldots, K}_{n-k-2}, E, E) d \lambda,
\end{aligned}
$$

which when rewritten gives the corollary. 
Theorem 1 and Corollary 2 to Theorem 2 give lower bounds for the volume of the relative inner parallel body $K_{-\lambda}$. Which inequality is sharper? For $n>3$ this has not been determined, but we will show that Corollary 2 is sharper when $n=3$.

When $n=3$, Theorem 1 is

$$
V\left(K_{-\lambda}\right) \geq V-\lambda S+2 \lambda^{2} W_{2}+\lambda^{2} W_{2}\left(K_{-\lambda}\right)
$$

and Corollary 2 to Theorem 2 is

$$
V\left(K_{-\lambda}\right) \geq V-\lambda S+\frac{3}{2} \lambda^{2} W_{2}+3 \int_{0}^{\lambda} t W_{2}\left(K_{-t}\right) d t
$$

By Lemma $3, W_{2}\left(K_{-t}\right)$ is a concave function of $t$. Thus for all $t$ satisfying $0 \leq t \leq \lambda$

$$
t W_{2}\left(K_{-t}\right) \geq t\left[\left(1-\frac{t}{\lambda}\right) W_{2}(K)+\frac{t}{\lambda} W_{2}\left(K_{-\lambda}\right)\right]=W_{2} t+\frac{1}{\lambda}\left[W_{2}\left(K_{-\lambda}\right)-W_{2}\right] t^{2} .
$$

This implies that

$$
\int_{0}^{\lambda} t W_{2}\left(K_{-t}\right) d t \geq \frac{1}{2} \lambda^{2} W_{2}+\frac{1}{3} \lambda^{2}\left[W_{2}\left(K_{-\lambda}\right)-W_{2}\right]=\frac{1}{6} \lambda^{2} W_{2}+\frac{1}{3} \lambda^{2} W_{2}\left(K_{-\lambda}\right) .
$$

Substituting the estimate in (11) into (10) gives

$$
V\left(K_{-\lambda}\right) \geq V-\lambda S+\frac{3}{2} \lambda^{2} W_{2}+3\left[\frac{1}{6} \lambda^{2} W_{2}+\frac{1}{3} \lambda^{2} W_{2}\left(K_{-\lambda}\right)\right],
$$

which when simplified is (9). This shows that the right hand side of (10) is greater than the right hand side of (9), and therefore (10) is a stronger inequality.

In 1978 G. Matheron [9] showed the following:

Theorem 3. If $K$ is a convex body in $\Re^{2}$ with area $A$, perimeter $L$, and inradius $r$, then for all $\lambda$ such that $0 \leq \lambda \leq r$ one has

$$
A\left(K_{-\lambda}\right) \geq A-\lambda L+\pi \lambda^{2} .
$$

He conjectured that in $\Re^{n}(13)$ would extend to

$$
V\left(K_{-\lambda}\right) \geq \sum_{i=0}^{n}(-1)^{i} \lambda^{i} W_{i}(K)
$$

for all convex bodies $K$ in $\Re^{n}$ with inradius $r$ and for all $\lambda$ satisfying $0 \leq \lambda \leq r$. (14), if true, would be a "Steiner-like" formula for the volume of inner parallel bodies.

Sangwine-Yager showed in [12] that (14) does not hold in $\Re^{3}$. However, when $n=2$ and $E=B$ both Theorem 1 and Corollary 2 to Theorem 2 become Matheron's inequality (13). Therefore both can be viewed as extensions of (13) to $\Re^{n}$.

The following is an open conjecture, due to Teissier [14].

Conjecture. If $a_{1} \leq \ldots \leq a_{n}$ are the real parts of the roots of

$$
\sum_{i=0}^{n}\left(\begin{array}{c}
n \\
i
\end{array}\right)(-1)^{i} \lambda^{i} W_{i}(K),
$$

where $K$ is a convex body in $\Re^{n}$ and $\lambda \in \Re$, then $0<a_{1} \leq r \leq R \leq a_{n}$, where $r$ and $R$ are the inradius and circumradius of $K$, respectively. 
We mention this conjecture because it is related to Bonnesen's inequality, and because Wills is the only person to have made progress towards a proof (see [16]). We now consider the strengthenings of the Wills conjecture.

When $E=B$ and $\lambda=r$ Theorem 1 becomes

$$
\begin{aligned}
0 \geq & V-r S+(n-1) r^{2} W_{2} \\
& +(n-1)(n-2) \int_{0}^{r}(r-s) V\left(K_{-s}, \ldots, K_{-s}, K_{-r}, B, B\right) d s .
\end{aligned}
$$

Since mixed volumes are monotone and $r B \subseteq K$, we know that

$$
W_{2}(K)=V(K, \ldots, K, B, B) \geq V(r B, \ldots, r B, B, B)=r^{n-2} \omega_{n},
$$

which shows that (15) is a strengthening of the Wills conjecture.

Again letting $E=B$ and $\lambda=r$, Corollary 2 to Theorem 2 becomes

$$
0 \geq V(K)-r S(K)+n \sum_{i=0}^{n-2} \int_{0}^{r} t V(\underbrace{K_{-t}, \ldots, K_{-t}}_{i}, \underbrace{K, \ldots, K}_{n-i-2}, B, B) d t .
$$

Since $(r-t) B \subseteq K_{-t}$ and $r B \subseteq K$, we have

$$
\begin{gathered}
0 \geq V(K)-r S(K)+n \sum_{i=0}^{n-2} \int_{0}^{r} t V(\underbrace{(r-t) B, \ldots,(r-t) B}_{i}, \underbrace{r B, \ldots, r B}_{n-i-2}, B, B) d t \\
=V(K)-r S(K)+n \sum_{i=0}^{n-2} \int_{0}^{r} t(r-t)^{i} r^{n-2-i} V(B) d t \\
=V(K)-r S(K)+n \omega_{n} \sum_{i=0}^{n-2} r^{n-2-i} \int_{0}^{r} t(r-t)^{i} d t \\
=V(K)-r S(K)+n \omega_{n} \sum_{i=0}^{n-2} r^{n-2-i}\left[\frac{r^{i+2}}{(i+1)(i+2)}\right] \\
=V(K)-r S(K)+n \omega_{n} r^{n} \sum_{i=0}^{n-2} \frac{1}{(i+1)(i+2)},
\end{gathered}
$$

or

$$
0 \geq V(K)-r S(K)+n \omega_{n} r^{n}\left(\frac{n-1}{n}\right)
$$

which when simplified is the Wills conjecture.

We can see that (16) is another strengthening of the Wills conjecture, since

$$
V(\underbrace{K_{-t}, \ldots, K_{-t}}_{i}, \underbrace{K, \ldots, K}_{n-i-2}, B, B) \geq V(\underbrace{(r-t) B, \ldots,(r-t) B}_{i}, \underbrace{r B, \ldots, r B}_{n-i-2}, B, B),
$$

by the monotonicity of mixed volumes.

An additional strengthening of the Wills conjecture is a corollary to the following theorem. 
Theorem 4. If $E$ is a fixed convex body and $K$ is any convex body in $\Re^{n}$ with volume $V$, relative surface area $S$, relative inradius $r$, and $W_{i+1}\left(K_{-r}\right)$ denotes the relative $(i+1)$ st quermassintegral of the relative inner parallel body $K_{-r}$, then

$$
0 \geq V-r S+\left[n \sum_{i=0}^{n-2} \frac{i}{i+1}\left(\begin{array}{c}
n-1 \\
i
\end{array}\right) r^{i+1} W_{i+1}\left(K_{-r}\right)\right]+(n-1) r^{n} \omega_{n} .
$$

Proof. By Lemma $1, K \supseteq K_{-\lambda}+\lambda E$. Therefore we have

$$
S \geq S\left(K_{-\lambda}+\lambda E\right) \text {. }
$$

If we let

$$
S_{-\lambda}^{*}=S\left(K_{-r}+(r-\lambda) E\right)
$$

and

$$
S^{*}=S\left(K_{-r}+r E\right)=S\left(\left[K_{-r}+(r-\lambda) E\right]+\lambda E\right),
$$

then by (7) we have

$$
S^{*}=n \sum_{i=0}^{n-1}\left(\begin{array}{c}
n-1 \\
i
\end{array}\right) \lambda^{i} W_{i+1}\left(K_{-r}+(r-\lambda) E\right),
$$

and

$$
S\left(K_{-\lambda}+\lambda E\right)=n \sum_{i=0}^{n-1}\left(\begin{array}{c}
n-1 \\
i
\end{array}\right) \lambda^{i} W_{i+1}\left(K_{-\lambda}\right) .
$$

Therefore by (17) and (19), we have

$$
S\left(K_{-\lambda}\right) \leq S-n \sum_{i=1}^{n-1}\left(\begin{array}{c}
n-1 \\
i
\end{array}\right) \lambda^{i} W_{i+1}\left(K_{-\lambda}\right),
$$

and by (18) we have

$$
S_{-\lambda}^{*}=S^{*}-n \sum_{i=1}^{n-1}\left(\begin{array}{c}
n-1 \\
i
\end{array}\right) \lambda^{i} W_{i+1}\left(K_{-r}+(r-\lambda) E\right) .
$$

Again by Lemma $1, K_{-\lambda} \supseteq K_{-\lambda-(r-\lambda)}+(r-\lambda) E=K_{-r}+(r-\lambda) E$, and so $W_{i}\left(K_{-\lambda}\right) \geq W_{i}\left(K_{-r}+(r-\lambda) E\right)$ for all $i=1, \ldots, n$.

This fact, when combined with (21) and (20), gives us

$$
\begin{gathered}
S_{-\lambda}^{*}+S-S^{*}=S-n \sum_{i=1}^{n-1}\left(\begin{array}{c}
n-1 \\
i
\end{array}\right) \lambda^{i} W_{i+1}\left(K_{-r}+(r-\lambda) E\right) \\
\geq S-n \sum_{i=1}^{n-1}\left(\begin{array}{c}
n-1 \\
i
\end{array}\right) \lambda^{i} W_{i+1}\left(K_{-\lambda}\right) \geq S\left(K_{-\lambda}\right) .
\end{gathered}
$$

Integrating both sides of the above inequality from 0 to $r$ with respect to $\lambda$, we have

$$
\int_{0}^{r} S_{-\lambda}^{*}+S-S^{*} d \lambda \geq \int_{0}^{r} S\left(K_{-\lambda}\right) d \lambda=V
$$

by Lemma 2 . 
Another expression for $S_{-\lambda}^{*}$, by (7), is

$$
S_{-\lambda}^{*}=S\left(K_{-r}+(r-\lambda) E\right)=n \sum_{i=0}^{n-1}\left(\begin{array}{c}
n-1 \\
i
\end{array}\right)(r-\lambda)^{i} W_{i+1}\left(K_{-r}\right),
$$

and also

$$
S^{*}=S\left(K_{-r}+r E\right)=n \sum_{i=0}^{n-1}\left(\begin{array}{c}
n-1 \\
i
\end{array}\right) r^{i} W_{i+1}\left(K_{-r}\right)
$$

Substituting these expressions for $S_{-\lambda}^{*}$ and $S^{*}$ into (22), we have

$$
\begin{gathered}
V \leq \int_{0}^{r} S+n \sum_{i=0}^{n-1}\left(\begin{array}{c}
n-1 \\
i
\end{array}\right)(r-\lambda)^{i} W_{i+1}\left(K_{-r}\right)-n \sum_{i=0}^{n-1}\left(\begin{array}{c}
n-1 \\
i
\end{array}\right) r^{i} W_{i+1}\left(K_{-r}\right) d \lambda \\
=\int_{0}^{r} S+n \sum_{i=0}^{n-1}\left(\begin{array}{c}
n-1 \\
i
\end{array}\right)\left[(r-\lambda)^{i}-r^{i}\right] W_{i+1}\left(K_{-r}\right) d \lambda \\
=r S+n \sum_{i=0}^{n-1}\left(\begin{array}{c}
n-1 \\
i
\end{array}\right)\left(-\frac{i}{i+1}\right) r^{i+1} W_{i+1}\left(K_{-r}\right) \\
=r S-\left[\begin{array}{c}
\left.n \sum_{i=0}^{n-2}\left(\begin{array}{c}
n-1 \\
i
\end{array}\right)\left(\begin{array}{c}
i \\
i+1
\end{array}\right) r^{i+1} W_{i+1}\left(K_{-r}\right)\right]-n\left(\frac{n-1}{n}\right) r^{n} \omega_{n} .
\end{array}\right.
\end{gathered}
$$

This completes the proof.

Corollary 1. When $E=B$, the inequality

$$
0 \geq V-r S+\left[n \sum_{i=0}^{n-2} \frac{i}{i+1}\left(\begin{array}{c}
n-1 \\
i
\end{array}\right) r^{i+1} W_{i+1}\left(K_{-r}\right)\right]+(n-1) r^{n} \omega_{n}
$$

is a strengthening of the Wills conjecture since

$$
\left[n \sum_{i=0}^{n-2} \frac{i}{i+1}\left(\begin{array}{c}
n-1 \\
i
\end{array}\right) r^{i+1} W_{i+1}\left(K_{-r}\right)\right]
$$

is nonnegative.

How do these strengthenings compare to Osserman's strengthening (6)? Note that when $n=2$ and $E=B$, all four strengthenings become Bonnesen's inradius inequality

$$
0 \geq A-r L+\pi r^{2}
$$

We will first show that (15) is a sharper inequality than (6). If we apply Property 4 of mixed volumes to $V(B, K, \ldots, K, B)^{n-1}$, then we see that

$$
V(B, K, \ldots, K, B)^{n-1} \geq V(B, \ldots, B) V(K, \ldots, K, B)^{n-2},
$$

which is the same as

$$
W_{2}(K)^{n-1} \geq \omega_{n} W_{1}(K)^{n-2} .
$$

Since $W_{1}(K)=\frac{S(K)}{n}$, we have

$$
W_{2}(K) \geq \omega_{n}\left(\frac{S(K)}{n \omega_{n}}\right)^{\frac{n-2}{n-1}}
$$


If we rewrite (6) as

$$
0 \geq V(K)-r S(K)+(n-1) r^{2} \omega_{n}\left(\frac{S(K)}{n \omega_{n}}\right)^{\frac{n-2}{n-1}},
$$

then by (23) we can see that the right hand side of (15) is greater than the right hand side of (6), which means that (15) is a sharper inequality.

When $n=3$, Corollary 2 to Theorem 2 is a stronger inequality than Theorem 1 for all $0 \leq \lambda \leq r$. Therefore (16) is a sharper inequality than (15) when $n=3$. In other words,

$$
0 \geq V-r S+\frac{3}{2} r^{2} W_{2}+3 \int_{0}^{r} t W_{2}\left(K_{-t}\right) d t
$$

is a stronger inequality than

$$
0 \geq V-r S+2 r^{2} W_{2}+r^{2} W_{2}\left(K_{-r}\right)
$$

Again, for $n>3$ it is not known which inequality is stronger.

Theorem 4 with $n=3$ is

$$
0 \geq V-r S+3 r^{2} W_{2}\left(K_{-r}\right)+\frac{8}{3} \pi r^{3}
$$

Since $K_{-r}+r B \subseteq K$ (Lemma 1 ), by the monotonicity of mixed volumes we have $W_{2}\left(K_{-r}+r B\right) \leq W_{2}$. Thus

$$
W_{2} \geq V\left(K_{-r}+r B, B, B\right)=W_{2}\left(K_{-r}\right)+r V(B),
$$

or

$$
r^{2} W_{2} \geq r^{2} W_{2}\left(K_{-r}\right)+\frac{4}{3} \pi r^{3} .
$$

Therefore (24) is a stronger inequality than (25), which in turn implies that (16) is the strongest of the four strengthenings of the Wills conjecture when $n=3$.

We have seen that Bonnesen's inradius inequality can be extended to higher dimensions in many ways. The extensions given here involve volume, surface area, terms with integrals of certain mixed volumes, quermassintegrals of inner parallel bodies, and the inradius. The Wills conjecture has been shown to be false for $n>2$ when the inradius is replaced with the circumradius; however, Bokowski and Heil [3] obtained a similar result with the inequality reversed. Chakerian [6] derived a Bonnesen-style inequality which yields a strengthened form of the isoperimetric inequality in two dimensions, but which does not involve volume and surface area in higher dimensions. For more information on Bonnesen-style inequalities and related open questions, see [13], pages 324-325.

\section{REFERENCES}

1. A. D. Aleksandrov, On the theory of mixed volumes of convex bodies, Mat. Sb. 3 (1938), no. 45, 28-46. (Russian).

2. J. Bokowski, Eine verschärfte ungleichung zwischen Volumen, Oberfläche und Inkugelradius im $R^{m}$, Elem. Math. 28 (1973), 43-44. MR 48:7119

3. J. Bokowski and E. Heil, Integral representations of quermassintegrals and bonnesen-style inequalities, Arch. Math. 47 (1986), 79-89. MR 88b:52008

4. G. Bol, Beweis einer Vermutung von H. Minkowski, Abh. Math. Sem. Univ. Hamburg 15 (1943), 37-56. MR 7:474f

5. T. Bonnesen, Les problèmes des isopérimetres et des isépiphanes, Gauthier-Villars, Paris, 1929.

6. G. D. Chakerian, Higher dimensional analogues of an isoperimetric inequality of Benson, Mathematische Nachrichten 48 (1971), 33-41. MR 44:4643 
7. V. I. Diskant, A generalization of Bonnesen's inequalities, Soviet Math. Doklady 14 (1973), no. 6, 1728-1731 (translation of Doklady Akad. Nauk SSSR 213 (1973), 519-521). MR 49:3688

8. H. Hadwiger, Vorlesungen über Inhalt, Oberfläche und Isoperimetrie, Springer, Berlin, 1957. MR 21:1561

9. G. Matheron, La formule de Steiner pour les érosions, Journal of Applied Probability 15 (1978), 126-135. MR 58:12874

10. R. Osserman, Bonnesen-style isoperimetric inequalities, American Math. Monthly 86 (1979), 1-29. MR 80h:52013

11. J. R. Sangwine-Yager, Bonnesen-style inequalities for Minkowski relative geometry, Transactions of the American Mathematical Society 307 (1988), no. 1, 373-382. MR 89g:52007

12. $ـ$ A Bonnesen-style inradius inequality in 3-space, Pacific Journal of Mathematics 134 (1988), 173-178. MR 89f:52032

13. R. Schneider, Convex bodies: The Brunn-Minkowski theory, Cambridge University Press, Cambridge, 1993. MR 94d:52007

14. B. Teissier, Bonnesen-type inequalities in algebraic geometry, Seminar on Differential Geometry (Princeton), Princeton University Press, 1982, pp. 85-105. MR 83d:52010

15. J. M. Wills, Zum Verhaltnis von Volumen zur Oberfläche bei konvexen Körpern, Arch. Math 21 (1970), 557-560. MR 43:3923

16. Minkowski's successive minima and the zeros of a convexity function, Monatsh. Math. 109 (1990), 157-164. MR 91f:52012

2-36-6 TAMA-CHO, FUCHU-SHI, TOKYO 183, JAPAN

E-mail address: b-noah@hoffman.cc.sophia.ac.jp 